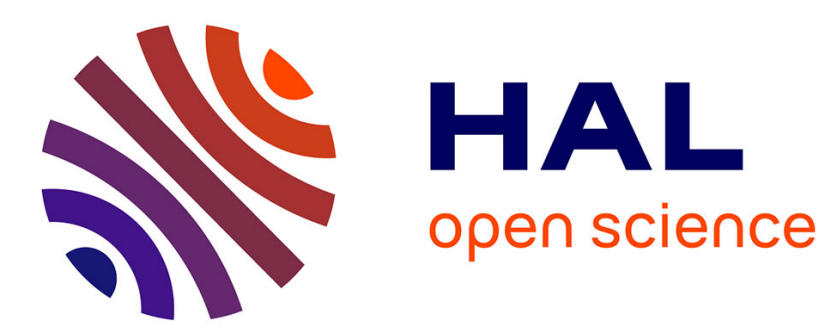

\title{
Urbanisation et développement: le cas de la Roumanie socialiste
}

Violette Rey

\section{To cite this version:}

Violette Rey. Urbanisation et développement: le cas de la Roumanie socialiste. Villes en parallèle, 1982, 6 (1), pp.10-24. 10.3406/vilpa.1982.944 . hal-02884388

\section{HAL Id: hal-02884388 \\ https://hal.science/hal-02884388}

Submitted on 29 Jun 2020

HAL is a multi-disciplinary open access archive for the deposit and dissemination of scientific research documents, whether they are published or not. The documents may come from teaching and research institutions in France or abroad, or from public or private research centers.
L'archive ouverte pluridisciplinaire HAL, est destinée au dépôt et à la diffusion de documents scientifiques de niveau recherche, publiés ou non, émanant des établissements d'enseignement et de recherche français ou étrangers, des laboratoires publics ou privés. 


\title{
Urbanisation et développement : le cas de la Roumanie socialiste
} Violette Rey

\begin{abstract}
Among the socialist countries, the dominion of the state reveale itself an incomparably keen instrument in the analysis of the urban network. The evolution of the distribution of the rumanian cities since the Second World War, is characterised by an improved spatial arrangement of the urban centers rather than, by transformations of the urban hierarchies. Thus in Rumania, in spite of the peculiarities of the rumanian population (demographic vitality, maintenance of a strong rural character), it is kept and affirmed the accordance in respect to the policy common to the east european countries, according to which, a regional equilibrium of the services related with a city is sought.
\end{abstract}

\section{Résumé}

Dans les pays socialistes, le cadre national se révèle un espace de référence incomparable pour l'analyse du réseau urbain. L'évolution du semis des villes roumaines depuis la deuxième guerre mondiale est moins caractérisée par des transformations de la hiérarchie urbaine que par une meilleure répartition spatiale des centres urbains. Ainsi, malgré les spécificités de la population roumaine — vitalité démographique, maintien d'une forte ruralité — s'affirme l'unité des politiques des pays d'Europe de l'Est pour un équilibre régional des services liés à la ville.

\section{Citer ce document / Cite this document :}

Rey Violette. Urbanisation et développement : le cas de la Roumanie socialiste. In: Villes en parallèle, $n^{\circ} 6$, octobre $1982 .^{\circ}$ Croissance et structures urbaines dans les pays socialistes européens. pp. 10-24;

doi : https://doi.org/10.3406/vilpa.1982.944

https://www.persee.fr/doc/vilpa_0242-2794_1982_num_6_1_944

Fichier pdf généré le 03/05/2018 


\section{RESUME}

Dans les pays socialistes, le cadre national se révèle un espace de référence incomparable pour l'analyse du réseau urbain. L'évolution du semis des villes roumaines depuis la deuxième guerre mondiale est moins caractérisée par des transformations de la hiérarchie urbaine que par une meilleure répartition spatiale des centres urbains. Ainsi, malgré les spécificités de la population roumaine - vitalité démographique, maintien d'une forte ruralité - s'affirme l'unité des politiques des pays d'Europe de l'Est pour un équilibre régional des services liés à la ville.

\section{ABSTRACT}

Among the socialist countries, the dominion of the state reveale itself an incomparably keen instrument in the analysis of the urban network. The evolution of the distribution of the rumanian cities since the Second World War, is characterised by an improved spatial arrangement of the urban centers rather than, by transformations of the urban hierarchies. Thus in Rumania, in spite of the peculiarities of the rumanian population (demographic vitality, maintenance of a strong rural character), it is kept and affirmed the accordance in respect to the policy common to the east european countries, according to which, a regional equilibrium of the services related with a city is sought. 


\section{URBANISATION ET DEVELOPPEMENT LE CAS DE LA ROUMANIE SOCIALISTE}

par Violette REY *

Dans les études géographiques récentes, le niveau national a souvent été retenu comme niveau d'analyse. Paradoxalement ce choix me paraît particulièrement fondé quand l'analyse a trait à l'Europe socialiste. A la fois, le très inégal état de développement atteint par chaque pays à la veille de la Seconde Guerre et la très forte planification centralisée de toutes les décisions et les activités depuis, tout comme le peu d'activités liées aux échanges hors des frontières, laissent, ou peutêtre renforcent, la personnalité de chacun, sous le couvert d'une référence à un modèle socialiste commun. C'est la raison pour laquelle je crois utile de caractériser à cette échelle le processus de la croissance urbaine récente, en tenant compte des travaux que les géographes roumains ont réalisé sur ce thème.

Deux ensembles de caractères généraux doivent êre rappelés dans la perspective de la croissance urbaine, l'un concerne l'état de la Roumanie, l'autre le système socialiste. La Roumanie est encore un pays fortement marqué par le fait rural; en 1948 elle n'avait que 3,7 millions de citadins, soit à peine le quart de sa population totale et les actifs agricoles représentaient $76 \%$ des actifs totaux; aucune ville ne dépassait 150.000 habitants, hormis la capitale Bucarest, qui jouissait par contre d'une position extrêmement dominante parce que déjà ville millionnaire. En outre, l'urbanisation et la volonté de croissance urbaine roumaine se réfèrent au modèle socialiste, qui considère l'urbanisation comme le processus contemporain le plus global en matière de restructuration sociale et d'élaboration de la société socialiste, une fois les infrastructures institutionnelles mises en place. Ce modèle d'urbanisation doit se développer en égal isant les situations régionales, en équilibrant la répartition des villes sur le territoire, afin que "les conditions de vie de la population rurale ressemblent toujours plus à celles des villes".

- Université de Paris I. 
Résultat effectif de ces trente années d'évolution, la population urbaine roumaine a atteint les 10,3 millions d'habitants, soit $48 \%$ de la population totale, les actifs agricoles représentent $33 \%$ des actifs, le nombre des villes est passé de 152 à 236 , avec comme projet d'atteindre les 505 unités en 1985. C'est sur le degré de réalisation de ce principe d'équilibre territorial - entre les villes et les campagnes, entre les villes entre elles - que je centrerai mon propos; je chercherai à évaluer dans la diffusion géographique de la croissance urbaine roumaine le degré d'homogénéisation territoriale acquis. L'approche se réfère seulement aux évolutions de répartition des villes selon leur taille; l'étude de la croissance en relation avec l'origine des citadins et avec les mouvements migratoires, tout comme celle de la croissance en liaison avec les activités dominantes, ne seront pas directement abordées dans cette communication. Je préciserai au préalable la manière dont la ville roumaine est définie dans les statistiques.

\section{I - La ville roumaine relève d'une décision législative}

Sans reprendre ici l'ample question de ce qu'est la ville, de ce qui mérite le nom de ville, je montrerai à travers les définitions adoptées par la Direction Centrale de la Statistique quelle finalité est attribuée à cette entité spatiale. Les localités roumaines et les aires territoriales qui leur sont administrativement attenantes sont actuellement classées en 3 types: les communes urbaines (villes et municipes), les communes suburbaines, les communes rurales. Chaque type est défini en fonction d'un complexe de critères, dans lequel le seuil usuel d'effectifs d'habitants ne joue qu'un rôle limité. La "ville» reçoit son statut juridique de ville par décision législative.

«Elle doit être un centre fortement peuplé doté de fonctions politiques et administratives, d'un potentiel industriel, commercial et socio-culturel significatif, et avoir de multiples liens avec sa région environnante sur laquelle elle exerce une influence économique et organisatrice»(1).

A ce statut s'attache donc un certain niveau d'équipement et d'autonomie de fonctionnement dans les deux domaines de la production et des services. La "commune suburbaine» est une commune de la proche périphérie d'une ville, qui a des liens de travail, transports et services, avec le centre, mais ne possède pas d'équipements socio-

(1) Plenum du CC du PCR du 5-6 octobre 1967, statuant sur l'amélioration de l'organisation administrative du territoire et de la systématisation des localités rurales. 
économiques autonomes. La "commune rurale» est en pratique définie par différence avec les précédentes. Une telle définition de la ville met en relief la conception retenue pour l'unité agglomérée comme un centre d'organisation sociale et de contrôle territorial. La mesure du processus de croissance urbaine sera donc étroitement liée à cette vision volontariste du législateur, ce qui n'est pas sans introduire certaines difficultés, puisque ne sont pas précisés par des chiffres les seuils utilisés pour chacun des critères démographiques, morphologiques et fonctionnels retenus. Mais cette imprécision même témoigne de la place exceptionnelle accordée à la ville, outil, agent de la transformation globale de la société en direction d'une complexité croissante des activités et des relations. La liberté laissée au législateur pour attribuer le statut de ville n'a pas pour autant transformé toutes les bourgades en des villes, qui doivent avoir ou recevoir les équipements correspondants. Par ailleurs, je rappellerai que tout contenu urbain reste relatif à une forme de développement et de structure socio-culturelle, et que dans le cas présent l'image de la ville roumaine n'exclut ni les basses densités d'habitat, ni une activité agricole parfois très importante. Ainsi, la densité urbaine moyenne était de 47 habitants à l'ha en 1974, et en 1976, 24 villes étaient classées agricoles parce que leur population active agricole était comprise entre 36 et $80 \%$ du total des actifs.

Les recensements exhaustifs de la population, principale source de documentation sur la croissance urbaine, ont eu lieu en 1930, 1948, 1956, 1966, 1977. Inévitablement tous ne relèvent pas des mêmes découpages territoriaux. En 1930 et 1948, la population urbaine départementale résidente comprend celle des villes et de quelques communes périphériques. De 1956 à 1965, elle comprend celle des villes et des 183 localités assimilées aux villes, selon la décision no 261 du conseil des Ministres. En 1966, une nouvelle décision a augmenté le nombre des localités assimilées à 238 . Le regroupement statistique dit de "l'unité urbaine" n'existe pas; la population de chaque commune urbaine est indiquée dans les Annuaires statistiques annuels. Enfin, il s'agit de la population résidente ayant un certificat de domicile pour la ville, ce qui exclut la population "flottante" qui habite plus ou moins durablement dans la ville considérée où elle a un emploi, sans en avoir le certificat de résidence. 


\section{II - Vers une réduction des disparités d'urbanisation}

C'est bien sur l'ensemble du territoire que l'urbanisation progresse et l'évolution des taux départementaux d'urbanisation montre une réduction progressive des écarts entre les départements les plus et les moins urbanisés. L'amplitude fut maximale en 1956, après la phase des fortes mutations sociales d'après guerre, et la réduction apparaît surtout sensible depuis 1966; en 1977, les taux oscillent entre $24 \%$ en Bistrita Nasaud et $72 \%$ dans le pays noir de Hunedoara. La carte de l'urbanisation (fig. 1) est un calque des zones de fort développement économique, avec principalement l'axe industriel central (du Timis au Prahova) et la zone du littoral et du Bas Danube. Par contre, la variation du taux d'urbanisation entre 1948 et 1977 montre au plan départemental une répartition tranchée, opposant parmi les départements dont l'urbanisation initiale était faible, ceux dont l'accroissement moyen a triplé et ceux qui n'ont pas encore atteint le doublement. Le premier groupe ceinture en une demi-couronne le foyer industriel central; l'autre correspond à la frange orientale du pays. En d'autres termes, cette carte suggère que l'égalisation des taux d'urbanisation s'opère selon une assez nette contiguïté spatiale, en diffusant de part et d'autre des noyaux urbanisés en 1948 (Brasov, Sibiu, Cluj, Ploesti).

Effet $d^{\prime}$ 'intenses mouvements migratoires, l'urbanisation roumaine a d'abord été le fruit de la forte fécondité de la population roumaine. D'où l'importance que l'on doit accorder aux comportements démographiques comparés de la population urbaine et de la population rurale dans chaque département, à la fois pour apprécier les stades d'évolution auxquels sont parvenues les différentes populations régionales et pour cerner le devenir de leur renouvellement démographique. Les quatre types de situations démographiques possibles existent effectivement (fig. 2). Un accroissement naturel nettement supérieur à l'accroissement départemental moyen aussi bien en zone urbaine que rurale est la caractéristique de la partie orientale du pays, dont j'ai précédemment souligné l'urbanisation modérée et en faible croissance; apparaît ici la forte fécondité moldave qui permet encore aux villages moldaves d'accroître leurs effectifs. A l'opposé, dans le type "banatique» occidental, les taux sont très faibles en zone urbaine (inférieurs à $5 \%$ ) et même négatifs en zone rurale; ce type commence à empiéter sur la Basse Valachie (département du Dolj et de Teleorman). Un troisième type est caractérisé par l'opposition entre un accroissement naturel élevé en zone urbaine et très bas en zone rurale, affectant le piémont valaque et deux départements montagneux de la Transylvanie occidentale. Ce type correspond à une urbanisation très récente; le passage de la campagne à la petite ville n'a pas encore ralenti la fécondité du type rural, mais les campagnes, ici depuis longtemps pourvoyeuses de nouveaux citadins pour l'ensemble de la Roumanie, n'ont plus pour elles-mêmes qu'une population vieillie et à excédent naturel très limité. Le quatrième type juxtapose deux 
Figure 1: raux d'urbanisation (d'aprés V.Cucu,1980)

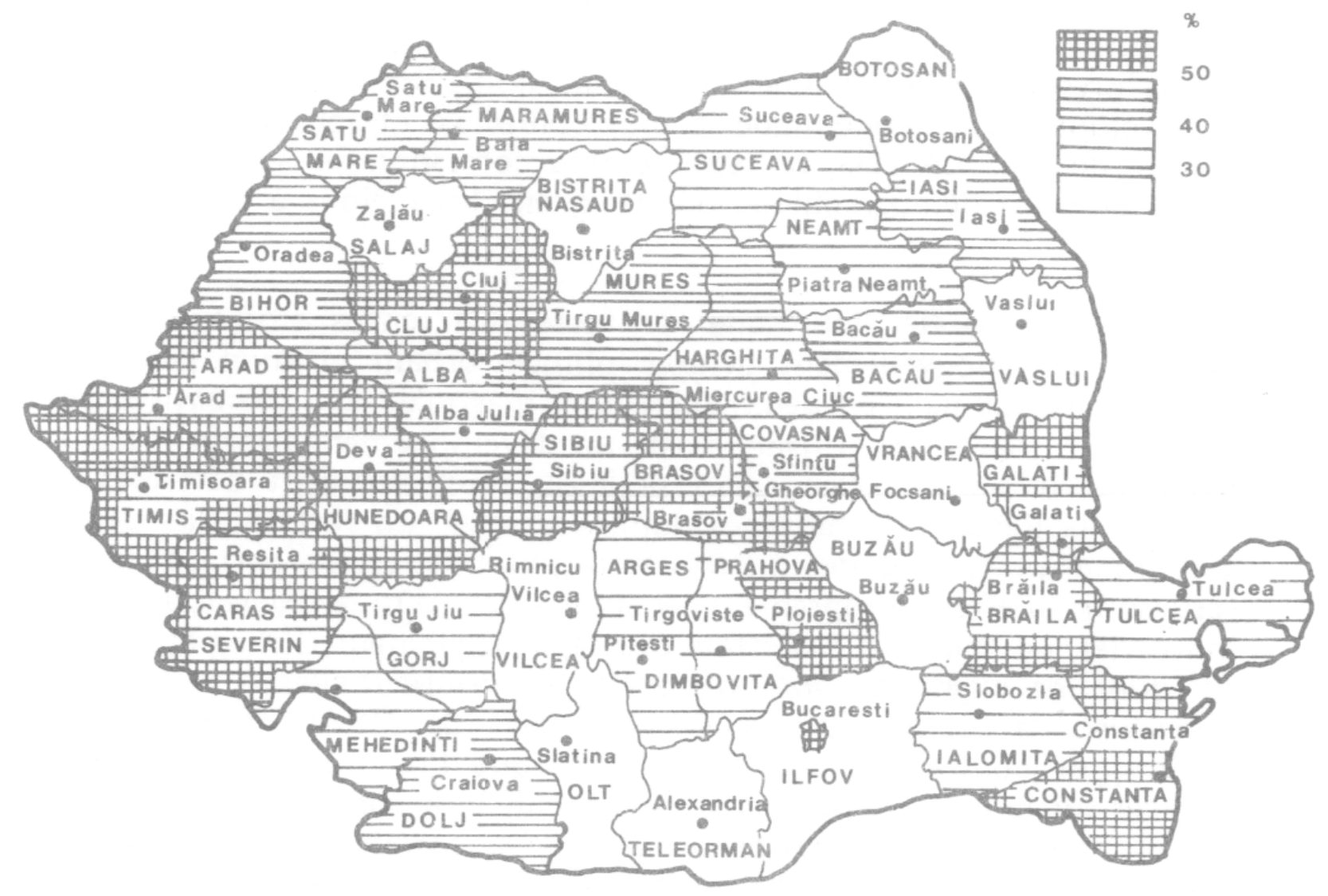

Fig. 2 Types departementaux de comportement démographique des populations urbaines et rurales_1977

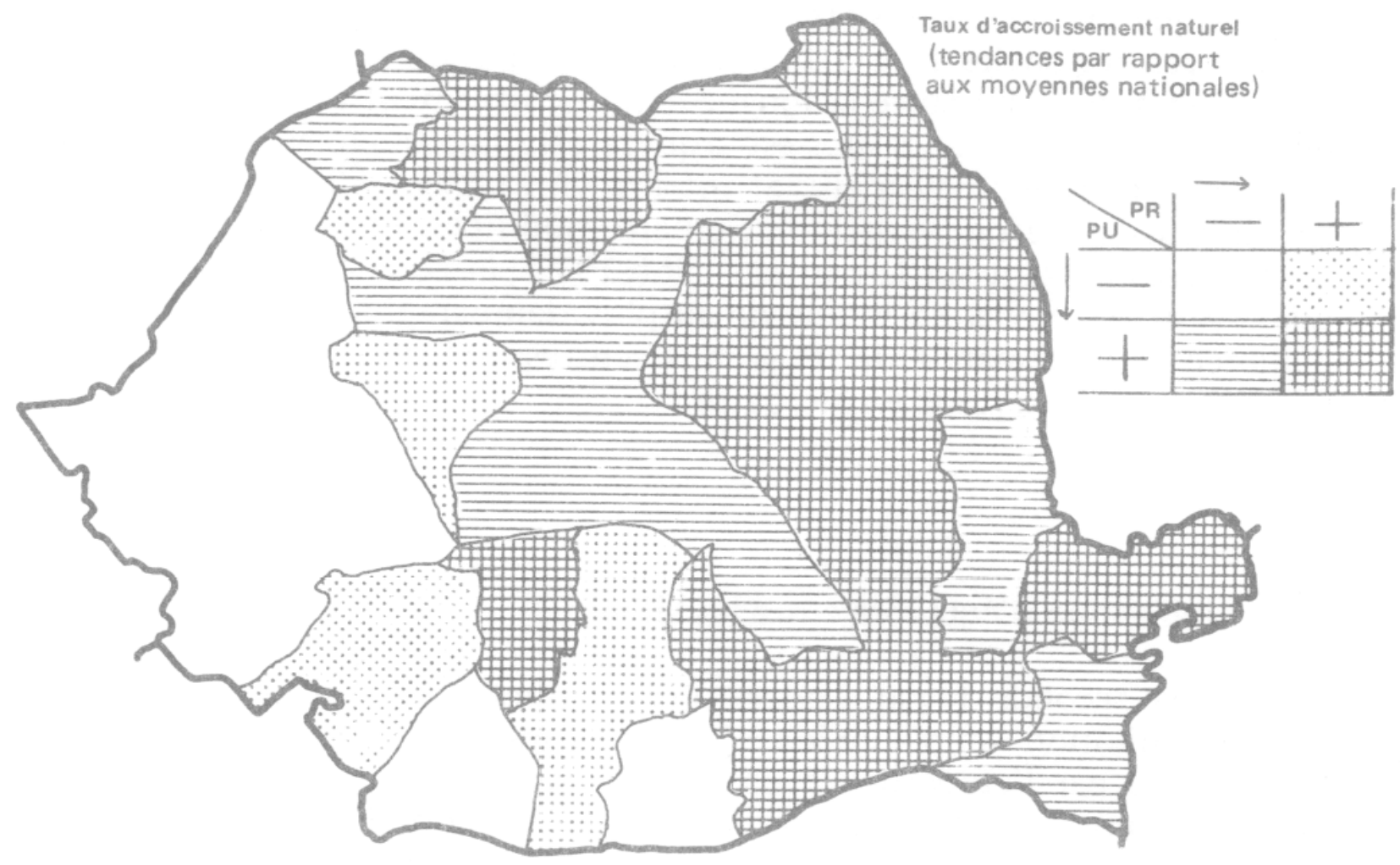


accroissements naturels modérés, légèrement inférieur au taux moyen urbain en zone urbaine, légèrement supérieur au taux moyen rural en zone rurale. Ce type correspond aux départements anciennement marqués par la présence d'une grande ville (Cluj, Sibiu, Brasov...), ayant atteint le développement économique le plus complexe et souvent le meilleur niveau de vie. En cela, ce type semble correspondre à la forme de comportement démographique qu'atteignent les régions à urbanisation évoluée.

On constate donc que de forte différences structurelles en matière de renouvellement démographique urbain et rural partagent le territoire roumain en deux, selon une diagonale approximativement NO-SE : à l'ouest de la ligne, existe un développement urbain marqué, mais inscrit sur des campagnes déjà ou prochainement désertées; à l'est de la ligne, les campagnes dominent, avec un fort surplus démographique porteur d'une urbanisation potentielle. Ce partage est la résultante d'effets proprement économiques dûs au développement industriel, combinés à des effets plus spécifiquement démographiques. En quoi les migrations inter-régionales contribuent-elles à réduire ou accentuer ce partage territorial? De l'avis de MEASNICOV (1977, p. 95), et contrairement à l'hypothèse que l'on pourrait facilement avancer sur la pression démographique rurale et la surpopulation agricole comme facteurs de migration à la ville, la corrélation entre le taux d'accroissement naturel global et le taux de départ hors du département est nulle $(r=-0,17)$, au moins jusqu'en 1966. Les Transylvains du Nord et les paysans valaques ont plus tôt et en plus grand nombre quitté leurs villages que les Moldaves. Cette observation met en valeur des différences d'attitudes régionales face à la mobilité, elle traduit vraisemblablement aussi l'effet de la loi entre la longueur du déplacement et le volume des départs, puisque la Moldavie s'est trouvée être la plus éloignée des zones à forte expansion industrielle et urbaine des années 50-60. Toutefois, les conclusions seront vraisemblablement modifiées, quand on connaîtra les analyses portant sur la période 1966-1977; la zone centrale occidentale ayant de moins en moins de surplus démographiques susceptibles d'alimenter la demande de ses grands centres industriels, son attraction migratoire portera jusqu'en Moldavie.

\section{III - Une action d'équilibre entre les villes}

La seconde façon d'approcher la dynamique de la répartition de l'urbanisation et d'apprécier l'effort consenti en matière d'équilibrage passe par l'étude de l'évolution des villes elles-mêmes, évolution mise en rapport avec le double objectif politique de l'aménagement du 
territoire : augmenter le nombre de villes plutôt que de laisser croître la taille des grandes villes, en assurer la meilleure répartition spatiale.

En matière de nombre de villes et de hiérarchie de tailles entre elles, la croissance urbaine contemporaine a réussi à ne pas accentuer l'écrasante primatie de la capitale mais à la réduire légèrement et elle a assuré l'apparition d'un nombre important de nouvelles unités. Bucarest, avec 1.060 .000 habitants en 1948 , concentrait $28 \%$ de la population urbaine du pays; en 1977, ses 1.934 .000 habitants n'en représentent plus que $19 \%$ (à la différence de l'évolution de Budapest, qui atteint $40 \%$ de la population urbaine hongroise). Quant au nombre de villes, il passe de 152 à 236 entre les deux dates, alors que stagnent ou même régressent les effectifs de villes dans les démocraties socialistes du Nord (Pologne, RDA...) ; ces mouvements sont à rattacher à l'état antérieur de chaque réseau national. En matière de croissance spécifique selon les tailles, il faudrait suivre les évolutions des villes en fonction de leurs tailles initiales (en 1930 ou en 1948), pour évaluer comment l'action d'incitation et de contrôle de la croissance a pu tenir compte de la taille moyenne souhaitée.

Le graphique dit de Zipf, ordonnant les villes (fig. 3) selon leur rang et leur taille, permet une appréciation des tendances spécifiques entre 1930 et 1977 . Le tracé de 1977, parallèle à celui de 1930, montre qu'en Roumanie pas plus qu'ailleurs la modification de la hiérarchie n'a été très forte, malgré l'ampleur de la mutation politico-économique intervenue depuis 1945 (1). L'inertie de la forme d'une répartition rang-taille à l'intérieur d'un système urbain national est donc toujours très importante. La similitude des tracés aux deux dates, entre lesquelles s'inscrit pourtant un triplement de la population urbaine, signifie donc que la quantité de nouveaux citadins roumains a été sensiblement proportionnelle à la taille initiale de chaque ville, et que les taux de croissance sont en moyenne identiques et indépendants des tailles. Ceci n'exclut pas certaines redistributions internes de rang, comme le montre le tracé des trajectoires (exemple de Brasov qui passe du 11e au 4e rang), ni le renforcement relatif de la part des grandes villes, tandis qu'un certain nombre ont subi un recul dans la catégorie des villes moyennes. C'est donc plus du côté de la trame spatiale que de la forme de la hiérarchie que sont à rechercher les modifications spontanées ou volontaristes introduites dans la croissance urbaine.

La transformation spatiale volontaire du semis de peuplement est-elle paradoxalement plus facile que l'infléchissement de la hiérarchie

(1) Voir à ce sujet D. PUMAIN. 1980 "Contribution à l'étude de la croissance urbaine dans le système urbain français», thèse d'E tat Université de Paris I. 
J

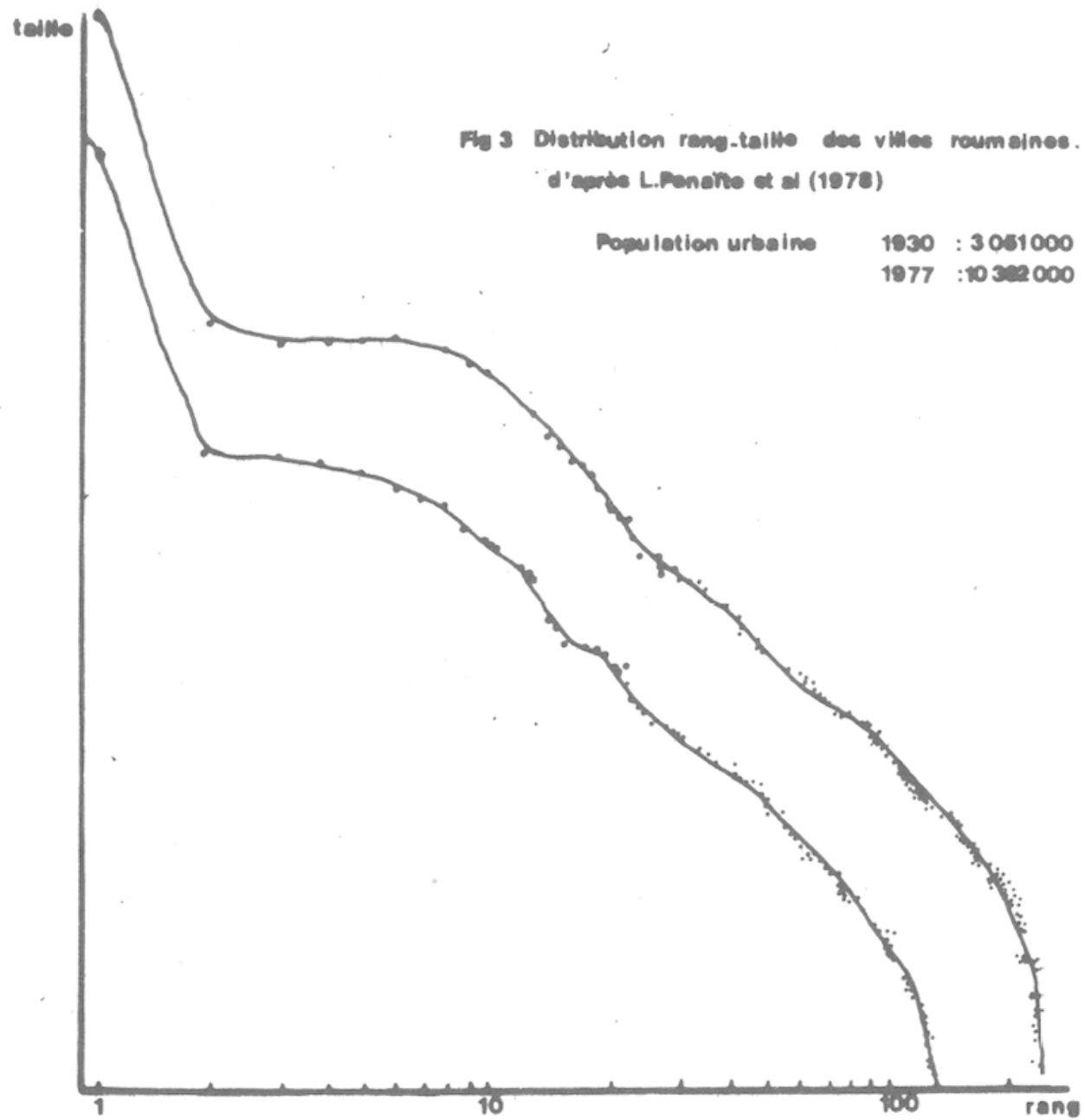


fig. 4 EVOLUTION DE LA TRAME URBAINE DE ROUMANIE

A. Trame urbaine pré-industrielle à semis régulier.

1. Situation en 1912.

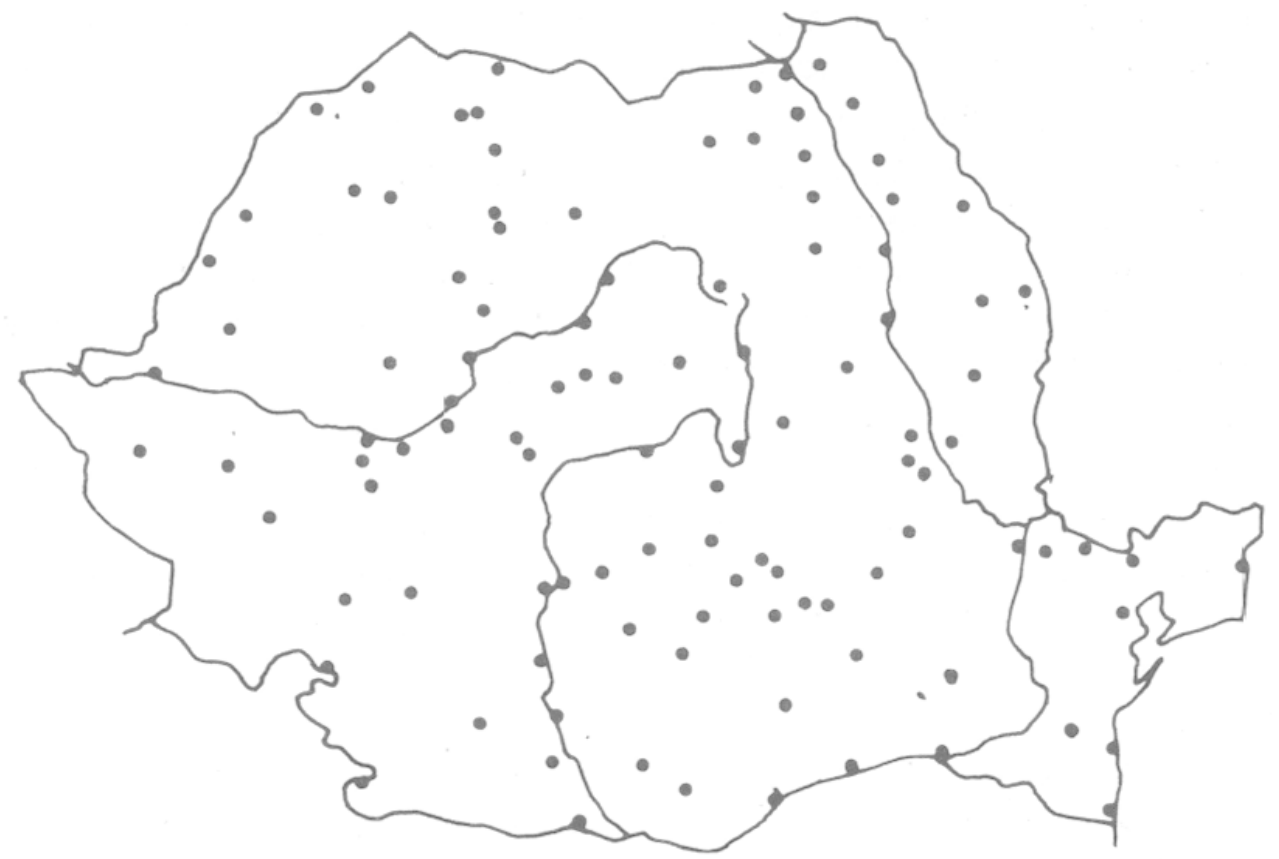

B. Urbanisation de type industriel à semis discontinu et concentré

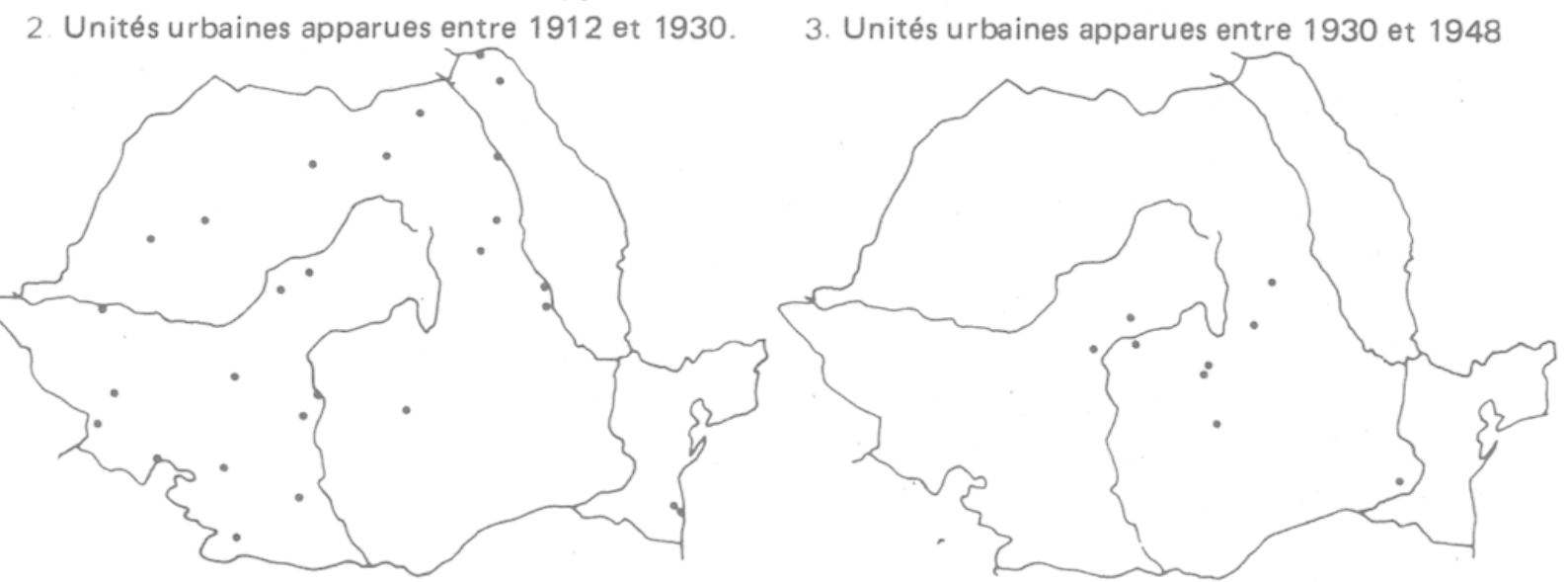

C. Urbanisation des campagnes avec régularisation du semis.

4. Unités urbaines apparues entre 1948 et 1966.

5. Unités urbaines entre 1966 et 1975
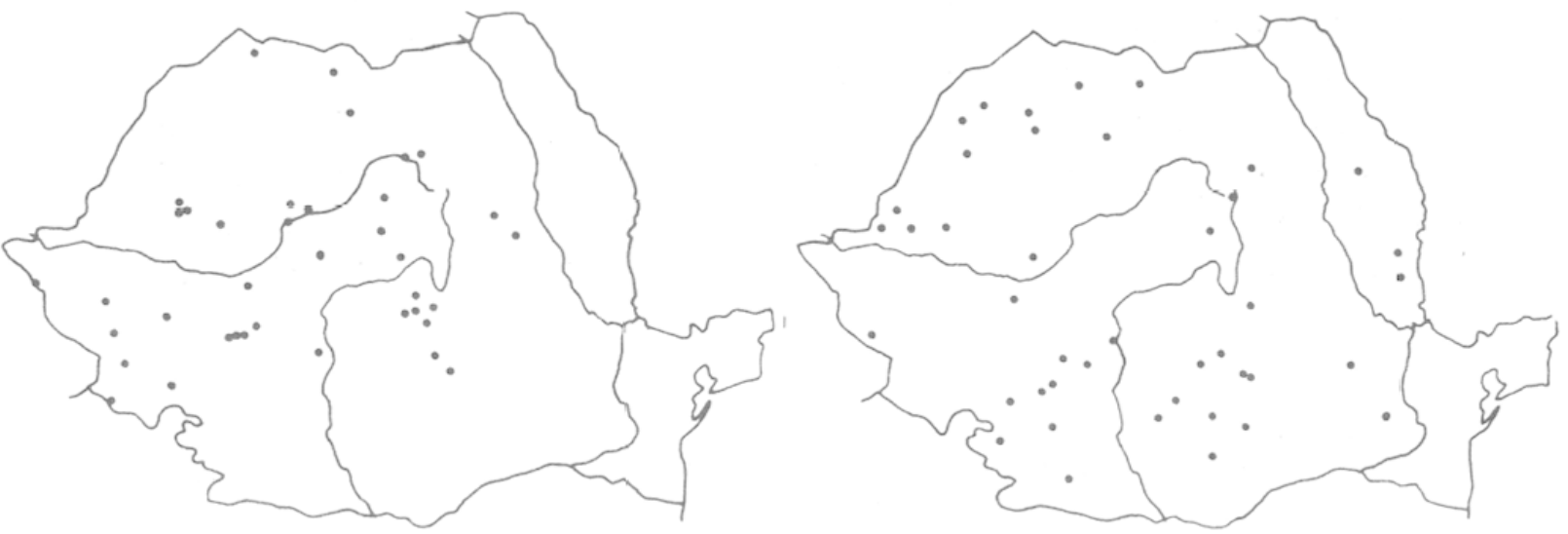
des tailles ? Alors même qu'on touche là à l'implantation physique des villes, donc à un élément incommensurablement plus fixe que les populations elles-mêmes. Là encore l'évaluation d'une transformation de ce type ne peut être correctement tentée que sur une durée suffisamment longue. J'ai donc cherché à insérer la volonté actuelle d'équilibre de répartition territoriale des villes dans I'histoire du réseau urbain roumain, en détachant les trames successives des nouveaux établissements urbains apparus depuis 1912 (1). De la cartographie de ces étapes, on peut dégager les conclusions suivantes (fig. 4). En 1912 (à un moment où l'unité roumaine n'était pas encore achevée), la régularité de la distribution l'emporte, même si la ligne de contact des piémonts transparaît, même si la densité est moindre dans les basses plaines le long du Danube ou à la bordure de la plaine hongroise. II s'agit d'un réseau urbain pré-industriel typique, où les villes, lieux d'échanges avec leurs campagnes environnantes, sont assez uniformément espacées, compte tenu des déviations introduites par l'agencement du relief. Entre 1912-1930, 27 nouveaux centres apparaissent surtout à l'ouest de l'Olt et le long du Siret, et ils assurent une amélioration de la régularité de répartition du semis. La rupture de cette tendance remonte aux années $1930: 8$ des 9 villes apparues entre 1930 et 1948 sont concentrées dans le triangle entre Sibiu, Ploesti, et Onesti. L'émergence du cœur économique du pays au niveau de la courbure carpatique, à cheval sur la Transylvanie du Sud et la Munténie s'individualise. La localisation de la quarantaine de créations effectuées entre 1948-1966 lors de la première phase d'industrialisation socialiste est précisément dictée par la présence des gisements à mettre en valeur : les zones montagneuses renforcent leur avantage; le semis des nouvelles unités a la forme bien particulière des urbanisations industrielles en grappe ou en chapelet, autour des gisements (Petrosani) ou le long de certaines vallées (Prahova). A partir de 1966, ce potentiel d'urbanisation spécifique est à peu près complètement mis en valeur (avec parfois même des régressions de petits centres miniers qui n'avaient d'urbain que le nom). II y a retour à un semis plus banal de centres promus au rang de villes, choisis en fonction de leur coefficient local de centralité, aidés par la diffusion des ateliers d'industries légères, et dont la charte de systématisation du territoire a fondé politiquement la finalité en 1967. Leur répartition reste cependant relativement spécifique puisqu'elle ne touche que les deux ensembles du N.O. (bassins du Somes et du Cris) et du Sud (Valachie), dont j'ai souligné la situation démographique en voie de vieillissement-appauvrissement et la moindre densité de villes dès 1912. L'effort certain de diffusion du fait urbain a donc porté sur les régions centrales et occidentales, où

(1) D’après l'Atlas national déjà cité $(1912,1930,1948,1966,1977)$ : les trois premiers intervalles sont de 18 ans et justifient pleinement la comparaison directe des cartes. 
la présence urbaine était plus forte, mais le support démographique plus fragile, avant de s'implanter sur la partie orientale du pays pourtant soumise à une très nette pression démographique rurale. Qu'en sera-t-il de la trame du lot de créations de 1980-85 ? Elle sera vraisemblablement localisée plus à l'Est, puisque le projet est que chaque département devra avoir au moins 7 villes en 1985, alors même que les densités globales oscillent de 30 à 174 .

La volonté d'équi-répartition territoriale systématique se traduit peut-être plus encore dans la hiérarchie fonctionnelle des centres que dans le simple semis. Dès 1973, j'en soulignais ce caractère dans un article des Annales (REY, 1973); et les cartes les plus récentes de hiérarchie urbaine confirment la stabilité de cette option (fig. 5). Les centres s'emboîtent régulièrement selon leur niveau hiérarchique et l'on souhaiterait faire quelques mesures pour vérifier la régularité de la configuration. Espacés sur l'ensemble du pays, les centres de premier ordre assurent l'armature urbaine du territoire. Les chefs-lieux de préfecture en constituent l'échelon suivant, et leur sélection comme chef-lieu en 1967 a parfois retenu la position géographique centrale dans l'unité administrative avant le poids démographique (exemple de Slobozia au lieu de Calarasi dans le département de Jalomita). La constitution de cette trame hiérarchique des centres est indissociable enfin de l'effort mené au niveau inférieur des bourgades rurales. La systématisation de l'habitat rural est en cours de réalisation, avec pour objectif de réduire le nombre de villages au profit des bourgs au centre d'unités communales agrandies. Cet aménagement est certainement un champ d'observation privilégié du mécanisme d'équilibrage territorial et du processus de transformation sociale par le biais de l'urbanisation.

Que conclure de ces premières observations en matière d'équilibre du réseau urbain ? Elles laissent la tentation d'identifier là une application du modèle de Christaller, fondé sur l'équi-répartition spatiale des centres de même niveau. Observée et conçue dans une économie capitaliste, cette configuration de trame paraissait à Christaller traduire de la manière optimale le principe du marché. Réalisée en économie socialiste, il y a substitution de la cause mais conservation de la forme territoriale : l'objectif d'égalité de desserte des "avantages" de la ville à l'ensemble de la population a remplacé celui du marché. Or ce souci majeur "d'équilibre dans la croissance urbaine» soulève certaines questions. Par quels moyens se réalise concrètement la croissance régulée ? Quelles réelles modifications des conditions du mode de vie sous-tendent la notion de progrès d'urbanisation ? En quoi un tel objectif est-il peutêtre plus adapté à une période de difficultés économiques générales que celui de la croissance des grands centres urbains ? Et surtout - question de fond devant laquelle achoppe tout aménagement du territoire jusqu'où le choix politique d'une équi-répartition des forces urbaines 
Figure 5 NIVEAUX DE HIERARCHIE DES CENTRES URBAINS d'aprés V.CUCU

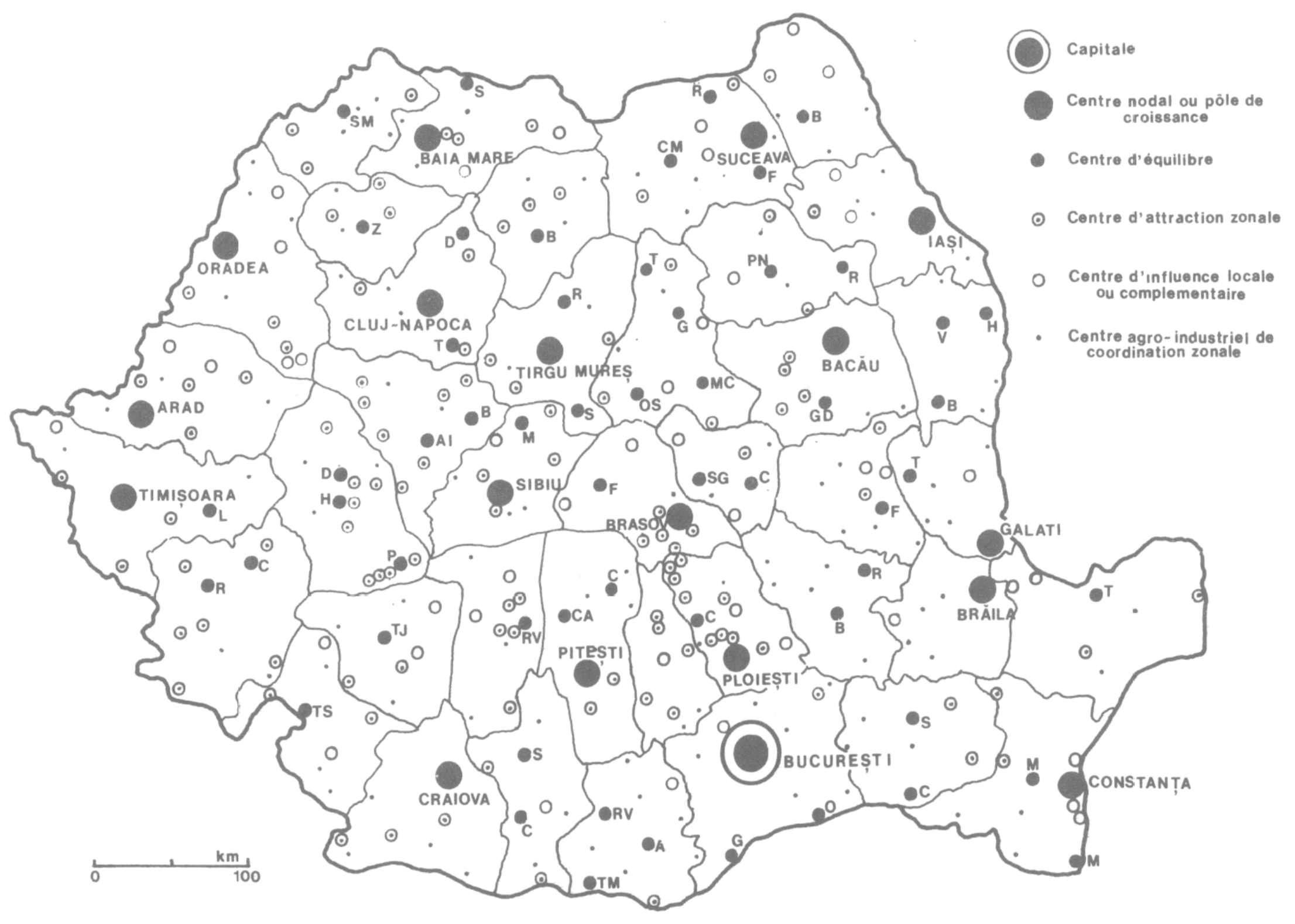


est-il compatible avec les économies d'échelle dont la grande ville serait porteuse ? Rappelons que l'amélioration générale des niveaux de vie a été retenue comme prioritaire; or elle passe par des gains de productivité, dont on sait qu'ils sont en partie produits par ces économies d'échelle.

De l'approche de la croissance urbaine, que j'ai appréhendée dans sa dimension quantitative, je retiendrai les conclusions suivantes. La forte ruralité et la vitalité démographique, dans lesquelles s'inscrit cette croissance, sont des marques spécifiquement roumaines. La volonté de réaliser une hiérarchie territoriale équilibrée et de contrebalancer le caractère dissymétrique du développement industriel par une diffusion des services est plutôt une spécificité de l'appartenance au système socialiste. Deux composantes beaucoup plus banales de l'évolution des systèmes urbains des pays à économie développée - en régime capitaliste ou socialiste - se sont révélées : I'homogénéisation des profils d'activité des villes qui accompagne leur développement et la place croissante du secteur tertiaire dans leur dynamique. Sans doute serait-il intéressant dans le cadre d'un groupe de travail sur la croissance urbaine en démocraties populaires d'établir une grille systématique de collecte des données urbaines; cela servirait à établir les "cartes d'identité" des croissances urbaines nationales, point de départ de travaux plus spécifiques sur les organisations urbaines. C'est dans cette optique que j'ai conçu la présentation ci-dessus.

Beaucoup d'aspects de la croissance urbaine roumaine n'ont pas été abordés ici. Or certains ont déjà fait l'objet de nombreux travaux; en particulier l'évolution de la morphologie intra-urbaine, l'évolution des aires d'attraction de main-d'œuvre et la desserte en transports, l'évolution d'une grande ville et/ou d'un réseau urbain régional, la mesure de certaines modifications introduites sur l'environnement par l'extension urbaine (en particulier des études de climatologie urbaine). D'autres aspects sont encore peu traités, alors qu'ils prennent une place croissante dans la littérature occidentale sur la ville; ainsi en est-il de l'étude du financement et des coûts de la croissance urbaine, de l'étude des "acteurs" qui concrètement participent aux politiques locales d'affectations territoriales (en zones à bâtir, zones de réserves territoriales, zones agricoles...), de l'étude de la nouvelle société urbaine dans sa dimension culturelle autant que sociologique. Les perspectives de travail sont donc multiples, et il sera sans doute fructueux de les envisager en collaboration avec les équipes roumaines travaillant sur le thème de l'urbanisation. 


\section{BIBLIOGRAPHIE}

J'ai limité cette bibliographie aux articles et ouvrages roumains récents les plus directement liés au thème de la croissance urbaine nationale et cités dans cette communication. Pour des analyses antérieures relatives à la géographie urbaine roumaine, je renvoie à REY $V$.

1. "Organisation régionale et structure urbaine de la Roumanie», d'après la littérature géographique roumaine récente, Annales de géographie, 1972, pp. 711.729.

2. "Brasov, une vocation urbaine», 1975, Bibliothèque Nationale.

3. "La Roumanie, essai d'analyse régionale», 1975, Paris SEDES. (RRGGG : Revue Roumaine de Géologie, Géophysique, Géographie).

APAVALOAIE M., LUPU BRATILOVEANU N.

"Les établissements industriels de RSR et leur influence sur la mobilité de la population» - RRGGG, 1980, pp. 155-160.

CONSTANTINESCU M, STAHL H.H., DRAGAN I.

"Urban growth processes in Romania", Meridiane Publishing house, Bucarest, 1974, $280 \mathrm{p}$.

Cucu V.

"Orasele Romaniei», Editura Stiintifica, Bucarest, 1970, 152 p.

CUCU V., DEICA P. "Le processus d'urbanisation en Roumanie», RRGGG, 1978, pp. 239-250.

IANOS I., OLARU M. "Contributions to the study of daily commutation in CarasSeverin Country», RRGGG, 1980, pp. 161-166.

MEASNICOV I, HRISTACHE I, TREBICI V.

"Demografia oraselor Romaniei», Editura stiintifica, Bucarest, 1977,223 p.

PANAITE L., CHITU M., CALOIANU N.

"First rank polarizing centres in Romanie's urban settlement network», RRGGG, 1978, pp. 251-256.

SANDRU I., CUCU V., POGHIRC P.

"Contribution géographique, classification des villes de la R.P. Roumanie», Annales de géographie, 1963, Paris, pp. 162 180.

STEFANESCU I. "Demographic types in Romanie and their geographical distribution», RRGGG, 1980, pp. 133-138.

TUFESCU V. "Sur l'urbanisation des villages de Roumanie», RRGGG, 1980, pp. 139-146. 